\title{
La eficiencia de la gestión del cambio en las empresas
}

\author{
The efficiency of change management in companies
}

Juan Carlos Sandoval Pérez. ${ }^{1}$, María Fernanda García Chuquimarca. ${ }^{2}$, Emilia Josefina Santamaría Cusco. ${ }^{3}$ \& Edwin Javier Santamaría Freire. ${ }^{4}$

DOI: https://doi.org/10.33262/visionariodigital.v5i4.1915

\begin{abstract}
Companies in competitive environments have the need to change quickly and adapt to change, becoming a characteristic of successful companies. Change management requires the implementation of change initiatives. The purpose of this research is to identify the factors that influence the effectiveness of change management in companies. For this, the behavior of 211 companies is observed, using a validity survey in search of business change initiatives that they carry as a tool to survive and prosper in a current competitive environment, they impact on the effectiveness of management. Of this, the elements stand out: awareness of the need for change promoting teamwork, senior management commitment, change in participation, change in communication, and change in motivation that help the planned change within the organization.
\end{abstract}

Keywords: Change Management; Organizational Communication; Participation; Effectiveness.

\section{Resumen}

Introducción, las empresas en entornos competitivos tienen la necesidad de cambiar rápidamente y adaptarse al cambio, convirtiéndose en una característica de las empresas

\footnotetext{
${ }^{1}$ Universidad Técnica de Ambato, Dirección Administrativa, Ambato, Ecuador, juansandoval@uta.edu.ec, https://orcid.org/0000-0002-0554-2269

2 Instituto Ecuatoriano de Seguridad Social, Dirección Provincial Tungurahua, Ambato, Ecuador. fersita77@gmail.com, https://orcid.org/0000-0002-7691-7646

${ }^{3}$ Universidad Tecnológica Indoamérica, Facultad de Ciencias Humanas y de la Salud, Ambato, Ecuador. esantamaria5@indoamerica.edu.ec, https://orcid.org/0000-0002-3268-1045

4 Universidad Técnica de Ambato, Facultad de Ciencias Administrativas, Ambato, Ecuador. edwinjsantamaria@uta.edu.ec, http://orcid.org/0000-0003-2636-9685
} 
exitosas. La gestión del cambio requiere de la implementación de las iniciativas de cambio. Objetivo, identificar los factores que influyen en la eficacia de la gestión del cambio en las empresas. Metodología, a través de la metodología cuantitativa, se observa el comportamiento de 211 empresas, a través del uso de una encuesta validad en búsqueda de las iniciativas de cambio empresarial. Resultados, las empresas llevan como herramienta para sobrevivir y prosperar en un entorno actual competitivo, las mismas impactan en la eficacia de la gestión. Conclusión, se destacan los elementos: la conciencia de la necesidad del cambio promoviendo el trabajo en equipo, compromiso de la alta dirección, cambio en la participación, cambio en la comunicación y cambio en la motivación que ayudan al cambio planeado dentro de la organización.

Palabras claves: gestión del cambio, comunicación organizacional, participación, eficacia.

\section{Introducción}

En un entorno empresarial actual que cambia rápidamente, la única ventaja competitiva de las empresas parece ser la capacidad de adaptarse al cambio (Lawler y Worley, 2006). El cambio es universal una constante a través de las empresas del sector público y privado (Brunton \& Matheny, 2009). Las organizaciones son expuestas constantemente a escenarios que cambian de manera constante, casa uno de estos cambios permite que se puedan enfrentar a distintos entornos teniendo en base objetivos estratégicos (Pérez, et ál. 2016). Las empresas del pasado no se enfrentaban tradicionalmente a la competencia y dinamismo del mercado por lo tanto no tenían incentivos para cambiar su forma de hacer negocios. Esto ha cambiado desde que la competencia está surgiendo en los sectores administrativos. Las presiones del gobierno, las expectativas en los niveles de rendimiento, la necesidad de mejorar la eficacia se ha visto a muchas empresas participar en diversas iniciativas de cambio.

Los cambios se introducen ya sea para mejorar la eficacia o para adaptarse a los cambios externos (Carnall, 2007). La mayoría de los cambios implementados en las empresas están dirigidos a mejorar los servicios, la calidad, la racionalización de los procesos, lo que permite lograr los objetivos. El cambio organizacional se caracteriza por la introducción de un nuevo organizador y acuerdos de trabajo (Harwood y Giles, 2005). El cambio será siempre un impacto sobre las personas en las empresas positiva o negativamente. Los cambios pueden ofrecer oportunidades de crecimiento y desarrollo para algunos y amenazas de establecer nuevas relaciones, habilidades y actividades para otros (Keifer, 2005). El impacto negativo de cambiar los resultados en el cambio organizacional es que los esfuerzos no cumplan los objetivos propuestos. Esto puede provocar un desperdicio de recursos, tener baja productividad. Muchos investigadores indican una alta tasa de fracaso de un 70 por ciento para la mayoría de las iniciativas de cambio (Kotter, 2008). 
De esto se plantea como interrogante: ¿Cuáles son los factores que influyen en la eficacia de la gestión del cambio en las empresas?, que se responde a través de la hipótesis: La gestión del cambio eficaz mejora el nivel de desempeño de las empresas.

Hoy en día en las empresas se ha visto una creciente presión sobre el cambio como un factor crítico de éxito de estas (Blanchard, 2010). En un mundo dinámico el cambio en el lugar de trabajo es un tema crucial, porque el cambio se ve principalmente como una respuesta adaptativa por el sistema en calidad de conjunto a través de subconjuntos con funciones específicas para mantener un equilibrio con un entorno cambiante (O'Reilly y Tushman, 2008). Sin embargo, varios académicos coinciden en que la mayor parte de la iniciativa de cambio fracasa, pero difiere en la tasa de fracaso. Burnes (2004), menciona una tasa de fracaso de cambio del 60\% mientras que Kotter (2008), menciona un $70 \%$. En la actualidad el énfasis está en el proceso de introducir cambios en el contexto en el que se colocará el cambio (Williams y Williams, 2007). Para ello, se plantea el objetivo: Identificar los factores que influyen en la eficacia de la gestión del cambio en las empresas

\section{Marco Teórico}

\section{Cambio}

El cambio es la sustitución, modificación o transformación de un proceso o fenómeno (Burduş y Căprărescu, 2008), o en la opinión de Paton y McCalman (2000), es un proceso continuo de la confrontación, la identificación, la evaluación y la acción. El cambio es universal, un proceso, ya sea voluntario o no, presente en todos los ámbitos de nuestras vidas profesional y personal. Independientemente de sus causas, el cambio debe ser conocido, analizado y en la medida en que esto es posible, controlable. El conocimiento de este proceso es un requisito preciso para la gestión de alto rendimiento que garantice la competitividad y desarrollo de las empresas contemporáneas (Braica, 2013).

El tema de cambio Braica (2009), es una preocupación constante en la sociología de las organizaciones, quizás debido a la importancia que se da a la lentitud burocrática y la rigidez, o a cuestiones concretas de enfrentamientos incesantes por líderes empresariales y los ejecutores de reformas administrativas.

Para entender los mecanismos del cambio, así como la innovación dentro de una empresa hay que considerar la naturaleza construida del cambio. El cambio no es un fenómeno natural, es un tema que requiere investigación. Sin embargo, el cambio no debe entenderse como una crisis o incluso como un proceso de adaptación o desarrollo, sino más bien, como una manifestación de una multitud de mutaciones dentro de las empresas (Braica, 2013).

En el tiempo que nos toca vivir, el cambio es parte de la vida cotidiana; o, mejor dicho, el cambio es el signo distintivo de la vida actual. Al cambio se lo considera como todo aquello que se modifica en el entorno y está fuera de nuestro control, que llega a nuestro ambiente es el resultado de una innovación realizada (Ordóñez, 2010). Los procesos ocultos son un aspecto crucial del cambio empresarial y cuando no son explícitos, pueden 
bloquear incluso las mejores intenciones. La expresión procesos ocultos se usa aquí en el sentido de cualquier dinámica encubierta o inconsciente (Marshak, 2007).

Se define al cambio como un acto o proceso a través del cual algo se convierte en diferente. El cambio organizacional es el movimiento de una organización fuera de su estado actual hacia un estado futuro para aumentar la eficacia (George y Jones, 2009).

Desde la perspectiva empresarial el cambio no surge de manera espontánea si no por dos factores, primero los factores externos a la empresa, dentro de estos se puede considerar la competencia, el gobierno, leyes, entre otros, así como también se puede tomar en cuenta factores interno como el desempeño laboral, economía de la empresa dentro de estos elementos existe un tercero enfocado en el empleado de la empresa que rige en base a su nivel educativo, necesidades familiares y valores (Pérez, et ál, 2017)

Un evento de cambio ofrece un medio para analizar situaciones en desarrollo que especifican los mejores medios de comunicación tanto dentro como fuera del grupo de gestión (McCalman y James, 2008).

\section{Gestión}

La gestión tiene su razón de ser en la medida que busque alcanzar los objetivos empresariales y a la vez en la medida que sea entendida como un proceso. El hecho de que una gestión se desarrolle como un proceso se desenvuelve en base a las funciones gerenciales dentro de una organización se ven enfrentadas a ejercitar diferentes funciones interrelacionadas, las cuales teóricamente suceden en forma lógica (Mora, et ál, 2016).

La administración es una práctica que consiste en dirigir, organizar y desarrollar a las personas, la tecnología y los recursos financieros para alcanzar con eficacia los objetivos empresariales (Robbins y Stephen, 2012). La importancia de la gestión nunca debe estar con excesivo énfasis en el éxito de una empresa que se atribuya a una buena gestión. Las funciones claves de gestión incluyen planificar, delegar, comunicar con claridad, motivar a los empleados, adaptándose para el cambio y generar constantemente ideas innovadoras (Sidikova y Mashhura, 2011).

Una gestión de éxito en las empresas aparte de una estrategia ideada es la habilidad con la que aprovecha las oportunidades, generen un buen desempeño duradero, se adapten a las cambiantes condiciones comerciales y resistan los retos competitivos (Thompson y Strickland, 2012). Una buena gestión estructura un entorno laboral que fomenta el entusiasmo, ofreciendo posibilidades para que el personal desarrolle de modo que actualicen sus conocimientos y puedan responder al cambio (Cenzo, 2009).

\section{Gestión del Cambio}

El cambio organizacional genera una serie de procesos que permiten cumplir objetivos, así como también otorgar oportunidades de mejoras en la empresa, además la gestión de cambio puede desarrollarse de dos formas, primero de manera espontánea otorgando 
cambios rápidos, o como el resultado de procesos continuos que dan oportunidad a que el cambio se genere de manera leve (Martínez, et ál, 2018).

El cambio organizacional constituye una manifestación de la innovación que cada vez más se requiere llevar a cabo en las empresas para enfrentar la influencia sistemática de las fuerzas externas en la que la competencia, la globalización y el entorno tan dinámico existente las condicionan. Entre los aspectos de nivel interno que más influencia ejercen en la eficacia del cambio se encuentra el papel de la alta dirección, el recurso humano, los objetivos y cualidad del cambio, así como la medición del impacto (Barroso y Delgado, 2007). La gestión del cambio es una aproximación a la transición de los individuos, equipos y empresas a un estado futuro deseado; con el fin de contribuir a corto o largo plazo resultados positivos hacia la empresa (Forneron, 2021).

Los agentes de cambio pueden ser gerentes o no directivos, empleados de la organización o consultores externos. Según Segredo (2013), los agentes de cambio son personas que actúan como catalizadores y asumen la responsabilidad de gestionar las actividades de cambio en la organización. Las organizaciones pueden contratar servicios de consultoría externa para el asesoramiento y la asistencia si los esfuerzos de cambio son mayores. La ventaja de contratar a estos es que pueden realizar cambios más drásticos como ellos no tienen que convivir con las consecuencias después de la aplicación. La gestión del cambio no consiste en establecer nuevos modelos de gestión que a la postre resulten ser solamente teorías pasajeras, más bien consiste en aprovechar los cambios del entorno empresarial para el bien de la organización, por ello, las empresas no solo deben ser flexibles, sino que quienes las manejan deben desarrollar una aguda percepción para anticiparse a los cambios y poder estar así siempre a la vanguardia (Vértiz, 2008). La experiencia que una empresa viva un proceso de cambio permite reconocer que se trata de mejorar sus sistemas de gestión y sobre todo a comprender que su contribución es una de las llaves que permitirá abrir el futuro y crear una cultura empresarial que reconoce la importancia de vivir el cambio todos los días (Vecino, 2014).

En un sentido más simple, gestión del cambio es el proceso de ayudar a un cambio de persona, grupo y empresa. La gestión del mundo implica un esfuerzo para planificar el cambio y ejercer influencia sobre otras personas en el proceso. Por lo tanto, la gestión del cambio implica un esfuerzo intencional para lograr un cambio (Martínez, et ál., 2018). Para Anderson y Anderson (2001) la gestión del cambio es visto como un conjunto de principios, técnicas y las prescripciones aplicadas a los aspectos humanos de la ejecución de las principales iniciativas de cambio en contextos empresariales. La gestión del cambio es parte de la gestión de recursos humanos ya que se ocupa de asesorar y asistir con la facilitación del cambio en las empresas en respuesta a los cambios del medio ambiente y la introducción de nuevas estructuras y sistemas organizativos, humanos, políticos y prácticas de recursos (Armstrong, 2005). Independientemente de la velocidad del cambio, la gestión del cambio es el movimiento de una empresa desde el presente existente hacia un estado futuro con el fin de aumentar la eficacia de las empresas (Cummings y Worley, 2005). La conducción motivadora para la gestión del cambio es el resultado de la necesidad de mejorar constantemente la productividad y la eficacia (Arnetz, 2005). 


\section{Planeación del Cambio}

El concepto de planeación del cambio parte de los aportes realizados por Lewin. Particularmente, de sus tres ejes teóricos (la teoría de campos, dinámicas de grupos, estudio de la acción y modelo de tres pasos para el cambio), que son relevantes para las empresas modernas al entenderlos de un modo integral (Burnes, 2006). A través del tiempo el concepto se ha desarrollado y aplica diferentes prácticas para que las empresas aumenten su efectividad (Karsten, 2009). Dentro del enfoque en el cambio se concentra la evaluación de la planeación del cambio y describe seis aspectos comunes, es decir, ser intencional, ser positivo, reflejar las circunstancias, centrado en los valores de la organización, orientación a la acción y que se enfoque en el aprendizaje (Okhuysen, 2001).

Los cambios pueden o no ser planeados. Cambios en la administración de impuestos podrían surgir en forma reactiva en respuesta a problemas externamente impuestos como cambios legislativos en busca de un proactivo mejoramiento de la eficiencia y eficacia (Bridges, 2009). La planeación del cambio se basa en el supuesto de que es un proceso que se mueve de un estado fijo a otro a través de una serie de pasos pre planificados (George y Jones, 2009). Este es un paso fundamental porque ayuda a entender las sinergias, las motivaciones, los riesgos los puntos débiles de la organización y las medidas para garantizar el éxito en las empresas (Aguilar, 2015).

Para asegurar una buena planeación del cambio menciona Manzano (2013) que se debe incluir en primer lugar un buen consultor interno o externo que facilite el proceso con experiencias y habilidades de comunicación e involucramiento. En segundo lugar, realizar un diagnóstico de la situación del cambio con sus fuerzas impulsoras y restrictivas. En tercer lugar, se construye el modelo del cambio con las variables que intervendrán y la manera como se relacionarán entre sí. En cuarto lugar, tiene que ver con los objetivos que se desean alcanzar una vez concluido el proceso. En quinto lugar, tenemos la ejecución, es decir, trazar la ruta crítica con acciones específicas para reducir las fuerzas restrictivas y fortalecer las fuerzas impulsadoras.

Un aspecto importante en la planeación del cambio es la participación de todas las personas involucradas en el proceso, las personas deben sentirse parte activa del cambio para poder comprometerse con las empresas (Orozco, 2014). La comunicación de todas las personas involucradas es fundamental y hoy en día las herramientas de comunicación vía internet juegan un papel importante para difundir los mensajes sobre lo que se está haciendo y lo que se desea lograr (Barranquero, 2013).

No podemos olvidar la importancia que da Senge (2000), a que los esfuerzos de cambio nacen en todos los niveles empresariales, se trata de encontrar aliados capaces de hacer realidad un cambio profundo que anime a comprender la única ventaja competitiva que la empresa del futuro tiene la capacidad de sus directivos a aprender a cambiar más rápidamente que la competencia. 
Poco éxito han tenido las estrategias propuestas por la literatura de planeación del cambio cuando no consideran a las personas. Las personas son un eje de estudio común (Nohria y Nitin, 2000). Lograr un cambio en los individuos es un requisito para impactar la organización observando la inercia desde la congruencia en las relaciones interpersonales, estudia las intervenciones formales que generan cambios incrementales ante lo requerido por el entorno (Okhuysen, 2001).

La manera de percibir estrategias de cambio va desde tres dimensiones actitudinales: cognitiva, emocional e intencional. La dimensión cognitiva trata de cómo se entiende y se perciben los aspectos de las empresas. La dimensión emocional trata la forma en que los individuos se sienten frente a éstas, mientras que la intencional, trata de lo que piensan hacer con éstas (Szabla, 2007).

Los Cambios se dividen en dos grandes categorías: el cambio evolutivo y el cambio revolucionario (George, 2009). La naturaleza del cambio influye en el nivel de complejidad, costo e incertidumbre que una empresa tiene que experimentar para abordar el cambio (Rees, 2013). Las teorías que utilizan estas dos categorías afirman que la vida en una empresa estará permeada por largos periodos de transformaciones incrementales puntuados por cortos periodos de fuertes transformaciones (Higgs, 2005).

\section{Cambio Evolutivo y Revolucionario}

El punto de vista evolutivo es primordial para comprender las características y el funcionamiento de cualquier sistema ya sea psicológico, social y cultural (Sala, 2001). Los cambios de estado, es decir, eventos o procesos son representables mediante una trayectoria que conecta dos o más puntos en el espacio de los estados dados. Y la historia total de un sistema es representable mediante la trayectoria completa que conecta el estado inicial del sistema con su estado final (Mahner, 2000).

Al existir cambio evolutivo contantes, las empresas buscan por medio de transformaciones de distintos nivele, otorgando beneficios que permiten que la empresa tenga una consistencia en el ámbito estratégico, estructural y cultural (Pérez, et ál ,2017). Según Van de Ven (2004), desde la perspectiva de la evolución natural, contingente al ambiente surge el equilibrio puntuado. En el equilibrio puntuado, las empresas fluctúan entre estabilidad y equilibrio y periodos revolucionarios. Además, se considera un patrón de variación, selección y retención similar a la teoría de ecología organizacional (Tsoukas, 2005).

Los tres elementos principales de la teoría del equilibrio puntuado son: primero las estructuras profundas, segundo el equilibrio de los periodos de evolución y tercero los periodos de revolución. Las estructuras profundas se consideran como elementos estructurales básicos que sostienen las empresas (Van de Ven, 2004). La evolución organizacional surge de distintas perspectivas del ser humano como fundamento, el cambio dentro de los seres humanos va desde distintas perspectivas por lo que se centra en una perspectiva socioeconómica (Lara, et ál, 2015). 
La existencia de cambio evolucionario eventualmente lleva a la formación de arquetipos dentro de las empresas. Un arquetipo es un set de estructuras y sistemas que reflejan un esquema interpretativo único, las cuales dan cohesión y consistencia a las empresas generando inercia, entendida como la resistencia al cambio (Jacome, et ál, 2018). Se generan dos tipos de inercia: la estructural y la cultural. La inercia más persistente es la cultural, pues representa la institucionalización de patrones de comportamiento por parte de los individuos. La inercia estructural es la resistencia al cambio causada por procedimientos y procesos (Romero y Campo, 2013). El cambio revolucionario puede definirse como el cambio de una configuración arquetípica a otra que altera radicalmente las practicas, para reflejar nuevos desafíos estratégicos (Amis, 2004).

\section{Importancia de la Comunicación en la gestión del cambio}

El entorno de las empresas modernas y sus procesos en el interior son muy dinámicos, estimulando el cambio constante de la empresa. Las empresas proporcionan importantes esfuerzos para la solución de problemas, pero no pueden evitar situaciones de crisis frecuentes. En la situación de las empresas cambiantes siempre hay una necesidad de volver a evaluar el proceso de gestión de crisis tanto a nivel teórico como a nivel práctico (Neville, 2005).

Los científicos Valackiene (2010) y Goodman (2006), señalan que los retos de la comunicación corporativa como una función de gestión estratégica están dados de la siguiente manera: la necesidad de construir la confianza con todos los clientes internos y externos; la experiencia con la corporación para lograr más con menos; la exigencia de construir una cultura corporativa global responsable y rendir cuentas en respuesta a un ambiente hostil para las empresas; la percepción ejecutiva de la comunicación corporativa como asesor del director general y gerente de la empresa; la comprensión del impacto global de la ley local y el impacto local de la ley a nivel mundial; la expectativa de que la empresa debe ser un buen ciudadano y ganar dinero; la comprensión de la transparencia como la mejor estrategia práctica para la gestión de la reputación y el conocimiento de que la comunicación corporativa sigue siendo a habilidad básica para la gestión.

Sin lugar a duda, la comunicación resulta ser una de las herramientas más importantes en la vida social y la gestión empresarial. También juega un papel fundamental en la colaboración de todas las actividades humanas que unen a la gente y crean relaciones (Duncan y Moriarty, 2008). Por otra parte, en el nivel de la organización Barker y Camarata (2008), afirman que la comunicación está integrada en todos los aspectos de convertirse en una organización eficaz. Como tal, la comunicación organizacional se ve esencialmente como el proceso iterativo que genera e interpreta mensajes entre las personas en las empresas a través de una dirección o varias direcciones (Stohl, 2005).

El análisis de la comunicación corporativa integra el planteamiento estratégico, el crecimiento del rendimiento de los procesos con el objetivo de aumentar el valor añadido para los clientes es uno de los objetivos básicos de las empresas modernas. El esfuerzo por mantener una posición competitiva en el actual entorno de mercado evoca una presión permanente en la mejora y el cambio en una empresa. La gestión del cambio en la empresa 
moderna es un requisito básico para una supervivencia en un entorno competitivo. Cada cambio debe ser anticipado y realizado eficazmente. Una condición necesaria para la realización exitosa de cambio es la gestión en temas de comunicación que juegan un papel importante. Por lo tanto, la gestión del cambio de una empresa debe ser considerada como un proceso de comunicación (Mazzei, 2010).

Muchos autores reconocen el papel facilitador que la participación empresarial tiene sobre los cambios. Barton (2002), califica la participación como un elemento que es especialmente importante entre las capacidades básicas de una empresa cuando se produce un cambio. Conger y Kanungo (2008), también sostienen que el sentimiento de participación es importante para estimular y direccionar cambios en las organizaciones, debido al hecho de que la incertidumbre asociada a los cambios genera un sentimiento de baja autoestima en los empleados; es aconsejable evitar tal sentimiento de baja confianza en sí mismo través de un programa participativo adecuado.

El papel del estilo participativo en la limitación de la resistencia al cambio es generalmente aceptado, afirmando que una tendencia a gestionar de una manera participativa aumenta la posibilidad de que se aceptara en cambio, así como la eficacia global de este cambio (Alutto y Belasco, 2002). De esta manera, los efectos principales que se atribuyen a un estilo participativo son doble, en primer lugar, se reduce la resistencia al cambio, y, en segundo lugar, ayuda a aumentar la eficacia.

La idea más general acerca de la resistencia al cambio es que las técnicas participativas son las mejores formas de gestionar la misma. La resistencia al cambio es uno de los temas clave en la gestión del cambio (Laframboise, et ál,2003). Los autores García (2011), confirman que el éxito del cambio solo es posible si se anticipan las principales fuentes de resistencia, porque el cambio siempre satisface a un grupo y perjudica a otro. Oyarvide, et ál. (2017), afirma que el compromiso y la participación son los factores más poderosos que la administración pueda utilizar para la aceptación del cambio, el autor presenta este tipo elementos debido a que ambos permitan dar un enfoque más allá del ámbito empresarial, enfocado hacia el quienes son y sus objetivos de a donde quieren llegar, esto permite que existan distintas formas de participación y compromiso.

La relación causal entre la participación y el compromiso se basa en la premisa de que más que los individuos puedan decidir sobre la forma y dirección que el cambio debe tomar, cuanto más se sientan implicados en el proceso de toma de decisiones, es decir, cuanto más participen, más probable será que van a estar comprometidos con el cambio. Con respecto a la relación entre el compromiso y la falta de resistencia por parte de los empleados, debe tenerse en cuenta que, cuando la responsabilidad para el cambio es compartida entre los ejecutivos y empleados este último no ve al primero como una figura de autoridad a revelarse en contra, lo que reduce cualquier resistencia que podría ser generada por este (Oyarvide, et ál, 2017). 


\section{Eficacia}

La eficacia es el conjunto de capacidades y habilidades para lograr determinados objetivos y metas. A mayor nivel de conciencia real y dominio de los conocimientos se tendrá mayores posibilidades de aplicar cualquier estructura empresarial con mayor eficacia (Lòpez, 2008). La eficacia mide la pertinencia de los objetivos y el grado en que la organización logra alcanzarlos. Las empresas son eficaces cuando los gerentes formulan objetivos apropiados para luego conseguirlos (Sanchez., 2010).

La eficacia indica el éxito en la consecución de objetivos. La evolución de la eficacia exige ajuste a los objetivos que se desean lograr a través de las metas que se perciben con antelación (Al-Hiyasat y Al-Shorafa., 2012).

La eficacia de la gestión fue conceptualizada en términos de competencia, satisfacción, resolución de conflictos, cumplimiento, valor de realización y reconocimiento (Srivastava y Sinha, 2007). Según Lehal (2000), la eficacia de gestión es reconocido como uno de los determinantes más importantes en las empresas que cambia rápidamente de ambiente.

\section{Metodología}

Dentro del estudio de las ciencias sociales se ha intentado seguir el paradigma positivista buscando las leyes que rigen a la realidad siguiendo esta base Max Weber pone énfasis en que cualquier acto realizado por el o los individuos posee una "carga" de intencionalidad propia, lo que supone la presencia de elementos subjetivos internos en el actuar de los sujetos. (Cárcamo, 2014). Así mismo siguiendo el camino popperiano la realidad será explicada a partir de leyes que pueden ser demostradas o falseadas en realidades particulares. Figueroa (2012) en base la realidad observada se compara con los conceptos teóricos estudiados a lo largo del tiempo y expuestos en el documento.

El trabajo de investigación busca determinar los factores que influyen en la eficacia de la gestión del cambio en las empresas, para lo cual requiere de información cualitativa, que lo define (Hernández, et ál, 2015), como descriptores de situaciones, eventos e interacciones observadas y sus manifestaciones.

Se define como población de estudio a las empresas pequeñas y medianas de la Provincia de Tungurahua, considerando que esta área geográfica concentra la mayor población por habitante (862 por cada 10.000 habitantes) del país, además el $90 \%$ de empresas son pequeñas y mediadas de acuerdo con cifras del censo económico 2010.

Para la muestra de estudio se considera 211 empresas que fueron seleccionadas aleatoriamente siempre que sean de la provincia y de tamaño pequeño o mediano, basándose en la base de datos de empresas registradas a las Cámaras gremiales.

La técnica de recolección de información se considera la encuesta, la misma que está diseñada en preguntas ordinales bajo escala de Likert de 5 niveles; el contenido del instrumento se divide en áreas o factores que influyen en el cambio, mostrado en la Tabla 1, la cual simplifica la recolección de información. Para validar el instrumento se aplica 
prueba piloto con 16 empresas participantes, donde se obtiene un Alfa de Cronbach de 0.838 indicando que el instrumento posee consistencia interna y el válido para su aplicación.

Tabla 1

Factores de cambio

\begin{tabular}{cc}
\hline Factor del cambio & Ítems preguntados \\
\hline Gestión del cambio & 4 \\
Comunicación corporativa & 4 \\
Gestión participativa & 4 \\
Desempeño empresarial & (utilidad generada) \\
Total & $\mathbf{1 3}$ \\
\hline
\end{tabular}

El procesamiento de la información incluye la transformación de la escala ordinal a proporción de modo que cada factor puede ser presentado como una proporción o probabilidad de existencia dentro de la empresa, es decir, los datos se transforman a cuantitativos, permitiendo la aplicación de técnicas estadísticas paramétricas.

\section{Resultados}

Como medida de existencia de estrategias formales de planeación del cambio se considera el plan estratégico como el medio donde se plasman las metas y objetivos de la organización que el $72 \%$ de empresas los mantienen; el 28\% de empresas no mantienen planes estratégicos en cuyo caso las acciones y metas se planifican basados en factores externos que no controla la empresa.

Figura 1

Planes estratégicos en la empresa

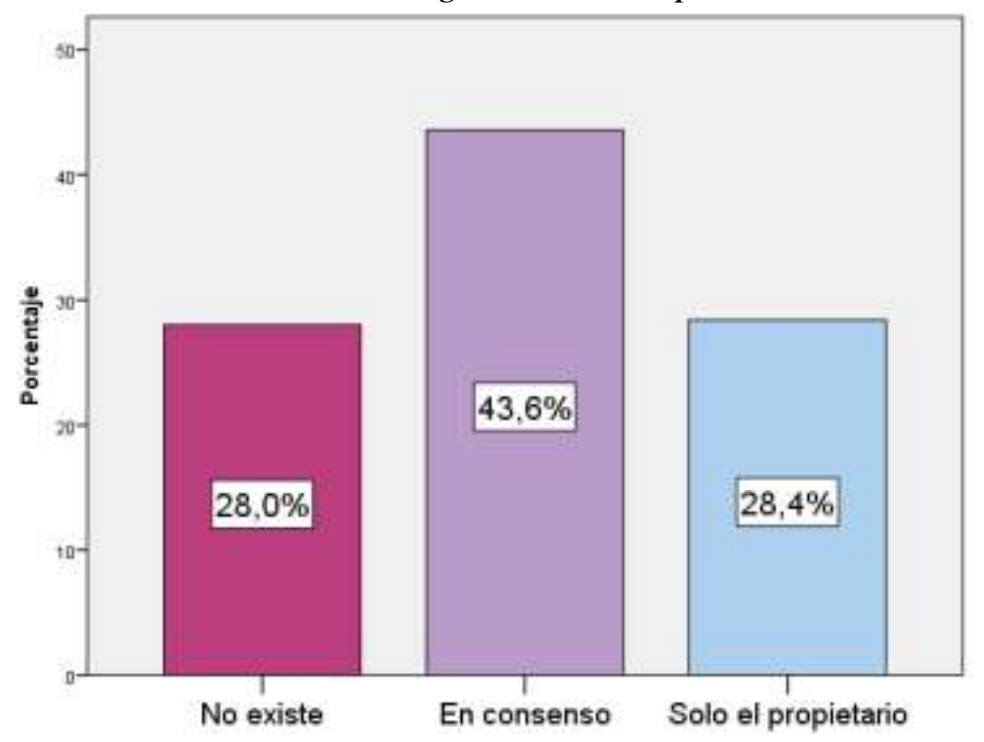

Del estudio realizado se encontró que las empresas difunden sus planes estrategias dentro de la organización, asegurando su cumplimiento. Al mismo tiempo se observa que las empresas pequeñas y micro mantienen dichos planes en reserva solo para conocimiento 
del grupo directivo y propietarios de la empresa. Esto ha creado que dichos planes sean más difíciles de ejecutar en la empresa y problemas de comunicación organizacional que hacen más complicado la transmisión de objetivos y metas.

\section{Figura 2}

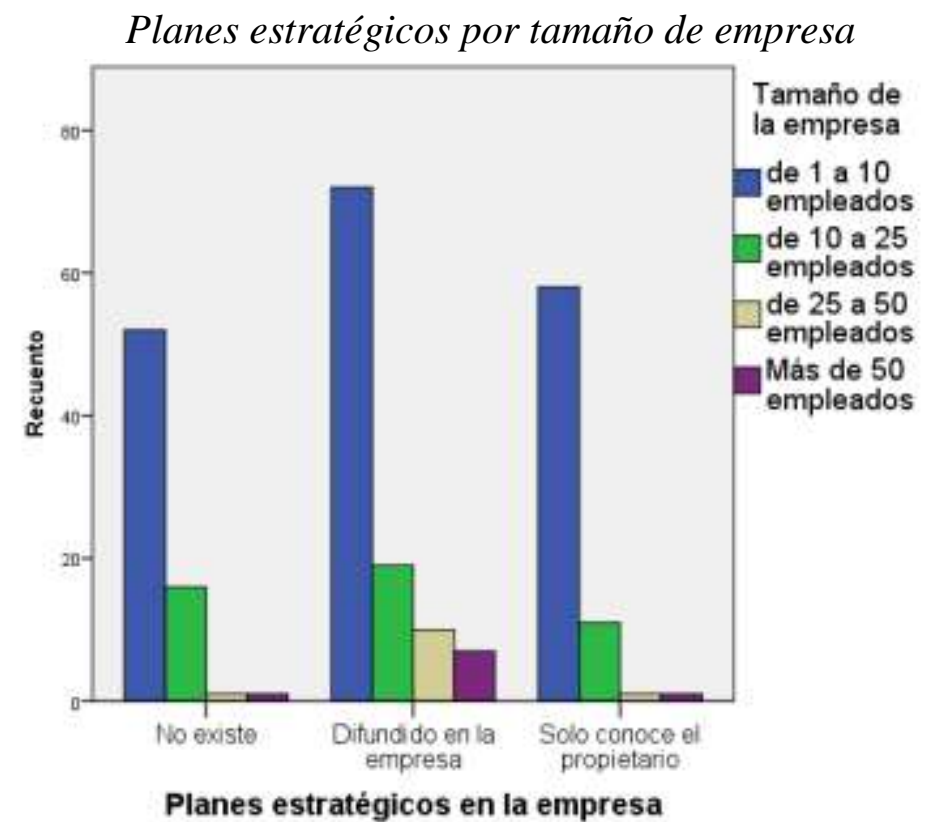

Para trasladar la planificación a la gestión la información pasa desde los planificadores hacia los actores de gestión del cambio, para ello se utilizan diversos medios como son la mayoría de los casos (46\%) mediante reuniones de trabajo, por lo general muy cercanas a las de planificación, por otra parte, el 32\% indica que la información de la gestión estratégica es abierta para los involucrados lo que permite que se realicen los trabajos adecuadamente.

\section{Figura 3}

De la planificación a la gestión estratégica

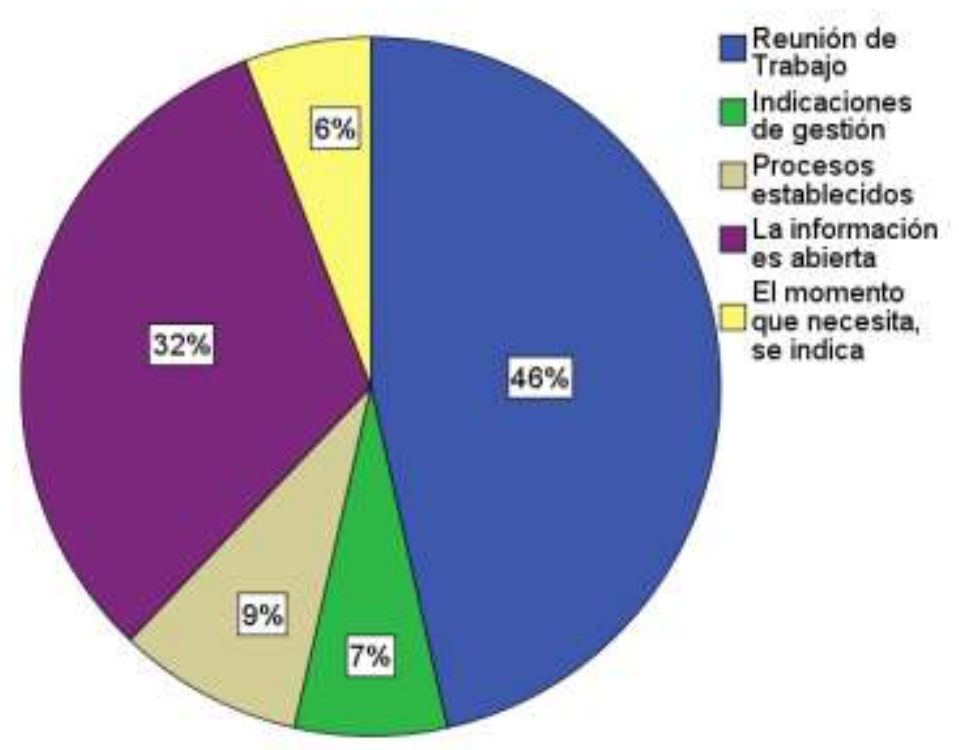


Para el control y verificación del cumplimiento de las actividades estratégicas se utilizan diversas herramientas que permitan verificar que los recursos invertidos están teniendo los resultados esperados. Siendo los informes y reportes los mecanismos de mayor uso en las diversas áreas de la empresa que resume diversos medios de recolección de información de la empresa, por su parte el reporte relaciona la revisión de información desde los históricos.

\section{Figura 4}

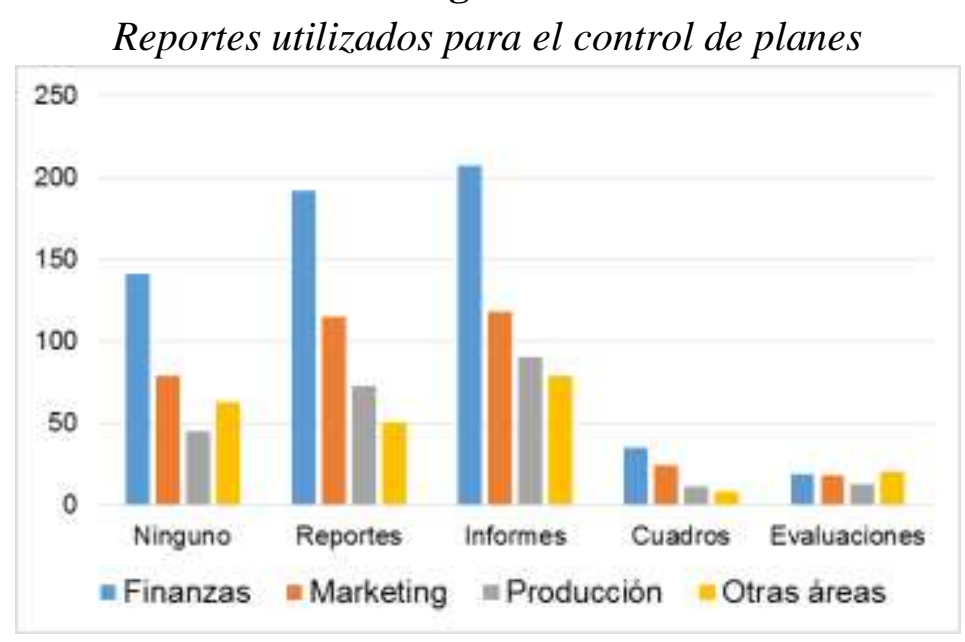

Para la medición de los factores se busca los nexos entre los elementos identificados, utilizando la correlación como medida de relación entre los factores. Expresados en la Tabla 2 donde es decir que hay evidencia que permite indicar que hay relación entre la gestión del cambio y la comunicación corporativa. Así mismo, hay relación entre la gestión del cambio y la gestión participativa en las empresas. Dichas aseveraciones con significación del 0.05

Tabla 2

Correlaciones de los factores

\begin{tabular}{|c|c|c|c|c|}
\hline & & $\begin{array}{c}\text { Gestión del } \\
\text { cambio }\end{array}$ & Comunicación & $\begin{array}{c}\text { Gestión } \\
\text { corporativa }\end{array}$ \\
\hline \multirow[t]{2}{*}{$\begin{array}{l}\text { Gestión del } \\
\text { cambio }\end{array}$} & $\begin{array}{l}\text { Correlación de } \\
\text { Pearson }\end{array}$ & & $0,477^{*}$ & 0,514 \\
\hline & Sig. (bilateral) & & 0,017 & 0,008 \\
\hline \multirow[t]{2}{*}{$\begin{array}{c}\text { Comunicación } \\
\text { Corporativa }\end{array}$} & $\begin{array}{c}\text { Correlación de } \\
\text { Pearson }\end{array}$ & $0,477^{*}$ & & $0,312^{*}$ \\
\hline & Sig. (bilateral) & 0,017 & & 0,023 \\
\hline \multirow[t]{2}{*}{$\begin{array}{c}\text { Gestión } \\
\text { participativa }\end{array}$} & $\begin{array}{c}\text { Correlación de } \\
\text { Pearson }\end{array}$ & 0,514 & $0,312^{*}$ & \\
\hline & Sig. (bilateral) & 0,008 & 0,023 & \\
\hline
\end{tabular}

Nota: *. La correlación es significativa en el nivel 0,05 (2 colas). 
Para la medición del desempeño se considera la cantidad promedio de carga transferida desde un estado de materia prima hasta construir un producto final en un tiempo determinado, Doerr y Sánchez, (2006) expresado por la diferencia entre la producción total y el consumo intermedio. Aguilar (2013) es decir, es la diferencia del valor de las ventas brutas y costo de producción. Por ello Zuleta, et ál. (2003), se considera la utilidad generada la expresión para el desempeño de las empresas, expresado en la Tabla 3, donde se observa el porcentaje de empresas que generan utilidad frente a la existencia de planes estratégicos en la empresa.

\section{Tabla 3}

Indicadores de desempeño

\begin{tabular}{ccc}
\hline INDICADOR & $\begin{array}{c}\text { Existe plan } \\
\text { estratégico }\end{array}$ & $\begin{array}{c}\text { No existe plan } \\
\text { estratégico }\end{array}$ \\
\hline $\begin{array}{c}\text { Valor agregado (utilidad neta de la } \\
\text { empresa) }\end{array}$ & $79.3 \%$ & $53.8 \%$ \\
\hline
\end{tabular}

A partir de lo cual se aplica test $\mathrm{t}$ de student, donde se encuentra $p$-value:0.005 evidencia de diferencias significativas entre los dos grupos de empresas, es decir que se demuestra que la gestión del cambio mejora el desempeño de las empresas.

\section{Conclusiones}

- La gestión del cambio esta vista como el proceso que se utiliza para ayudar a todos los interesados a adaptarse con éxito a los cambios que se están produciendo en las empresas, con el propósito de cambiar las partes interesadas de su estado actual a un estado futuro deseado. Es eficaz porque mejora el nivel de rendimiento en las empresas, el compromiso y apoyo de la alta gerencia que es una condición necesaria y suficiente para lograr los objetivos de esta.

- Los factores que influyen en la eficacia de gestión del cambio son: la conciencia de la necesidad del cambio promoviendo el trabajo en equipo, compromiso de la alta dirección, cambio en la participación, cambio en la comunicación y cambio en la motivación.

- En la actualidad, existen muchos desafíos y cambios, una empresa necesita la capacidad de responder rápidamente a dichos cambios. Con el fin de ser consistente y eficaz, las personas que laboran en las empresas necesitan información clara, pertinente y completa.

- La comunicación eficaz es la clave para mantener una empresa como un sistema de individuos que trabajan juntos por los objetivos de éxito.

- El vínculo que se estableció es que la participación conduce al compromiso y el compromiso evita la resistencia al cambio. Por lo tanto, muchos autores coinciden que uno de los más seguros métodos para superar la resistencia al cambio consiste en la participación de todas las personas en su diseño.

- Se determina que la gestión del cambio se puede evidenciar a través de la existencia de planes estratégicos en la empresa donde se sintetizan las acciones de cambio, comunicación y participación en los procesos empresariales. 
- Se encuentra relación entre la gestión del cambio, desempeño empresarial, comunicación corporativa y gestión participativa, actividades que son ejes de desarrollo de los planes estratégicos de las empresas.

- Las empresas están planificando constantemente, es decir, ante los factores externos y sus cambios, la reacción es la creación o actualización del plan estratégico que permite planificar y ordenar las actividades que serán el medio necesario para convertir las ideas en una acción trasformadora de la empresa. Por lo tanto, las actividades de cambio se originan como un cambio interno que es orientado siempre por un plan de trabajo que maximiza el esfuerzo de sus integrantes.

\section{Referencias bibliográficas}

Aguiar, V., Arghoty, A., Burgos, S., Gualavisí, M., Onofa, M., Ruiz, P., \& Saénz, M. (2013). Estudios industriales de la micro, pequeña y mediana empresa. Quito: Gráficas V\&M.

Aguilar, E. (2015). Importancia de una buena gestión del cambio. Mexico: Editora El Sol, S.A. de C.V.

Al-Hiyasat, \& Al-Shorafa. (2012). The Role of the Electronic Receipt of Public Revenues in Enhancing the Effectiveness of Control in the Public Sector in Jordan. Mu' tah Lil-Buhuth Wad-Dirasat: Mu'tah University.

Alutto, J., \& Belasco, J. (2002). A typology for participation in organizational. Administrative Science Quarterly, 117-25.

Amis, J. (2004). The pace, sequence, and linearity of radical change. Academy of Management Journal, 15 -39.

Anderson, \& Anderson. (2001). The Change Leaders' Roadmap: How to Navigate your Organization's transformation. San Francisco: Pfeffer.

Armstrong. (2005). A handbook of Human Resource Management Practice. London: Kogan Page Publishers.

Arnetz, B. (2005). Subjective indicators as a Gauge for Improving Organizational wellbeing, An attempt to apply the Cognitive Activation Theory to Organizations. Pschoneuroendocrinology, 1022-1026.

Barker, \& Camarata. (2008). The Role of Communication in Creating and Maintaining a Learning Organization. The Journal of Business Communication, 443-467.

Barranquero. (2013). Slow media. Comunicación, cambio social y sostenibilidad en la era del torrente mediático. Universidad de La Sabana, 419 - 448. 
Barroso, \& Delgado. (2007). GESTIÓN DEL CAMBIO ORGANIZACIONAL A TRAVÉS DE PROYECTOS. Instituto Superior Politécnico José Antonio Echeverría, 42 - 47.

Barton, L. (2002). Management of technology and moose on tables. Organization Science, $556-558$.

Blanchard, D. (2010). Supply Chain Managemen Best Practice. Estados Unidos: Wiley.

Braica, A. (2009). Managementul resurselor umane . University Press.

Braica, P. (2013). FUNDAMENTALS OF CHANGE MANAGEMENT. Economics Series, 1 -13.

Bridges, W. (2009). Managing Transitions. Philadelphia: Da Capo Press.

Brunton, \& Matheny. (2009). Divergent acceptance of change in a public health organization. Journal of Organizational Change Management, 19.

Burduş, E., \& Căprărescu, G. (2008). Androniceanu, A., Managementul schimbării. Brasil: Editura Economică, București.

Burnes, B. (2004). Emergent change and planned change - competitors or allies. International Journal of Operations \& Production Management, 886 - 902.

Burnes, B. (2006). Complexity theories and organizational change. International Journal of Management Reviews, 73 -90.

Cárcamo, H. (2014). Hermenéutica y Análisis Cualitativo. Cinta moebio 23, 204-216.

Carnall, C. (2007). Managing Change in organisations. Prentice Hall.

Cenzo, D. (2009). Fundamentos de Administración. Mexico, D.F.: Pearson.

Conger, J. A., \& Kanungo, R. (2008). The empowerment process: Integrating theory and practice. Academy of management review, 471-482.

Cummings, \& Worley. (2005). Organizational Development and Change. Mason: South Western College Publishing.

Doerr, O., \& Sánchez, R. J. (2006). Indicadores de productividad para la industria portuaria, Aplicación en América Latina y el Caribe. Santiago de Chile: Naciones Unidas.

Duncan, \& Moriarty. (2008). A Communication-Based Marketing Model for Managing Relationships. Journal of Marketing., 1 -13.

Figueroa, A. (2012). The Alpha-Beta Method Scientific Rules for Economics and the Social Sciences. Buenos Aires: Cengage Learning . 
Forneron, R. (2021). Gestión del cambio organizacional por covid-19 en las cooperativas de la ciudad de Pilar. Revista Multidisciplinar Ciencia Latina, 4914-4936.

García, Álamo, \& Hernández. (2011). Antecedentes de la resistencia al cambio: factores individuales y contextuales. Cuadernos de Economía y Dirección de la Empresa, 231-246.

George, \& Jones. (2009). Contemporary Management. New York: McGraw-Hill.

George, \& Jones. (2009). Contemporary Management. Burr Ridge: McGraw-Hill.

Goodman, M. (2006). Corporate Communication Practice and Pedagogy at the Dawn of New Millennium. An International Journal, 196-213.

Harwood, \& Giles. (2005). Interpersonal Communication. Boston: De Gruyter.

Hernández, R., Fernández, C., \& Baptista, P. (2015). Metodología de la Investigación. México: McGraw Hill.

Higgs, M. (2005). All changes great and small. Journal of change Management, 121 135.

Jacome, I., Tinajero, M., \& Suárez, I. (2018). La nueva administración del siglo XXI. Ciencias Económicas y empresariales.

Karsten, L. (2009). Leadership style and entrepreneurial change. Journal of Organizational Change Management, 73 - 91.

Keifer, T. (2005). Feeling bad: antecedents and consequences of negative emotions in ongoing change. Journal of Organisational Behaviour, 875-97.

Kotter, J. (2008). Choosing Strategies for Change. Harvard Business Review, 130-139.

Laframboise, D., Nelson, \& Schmaltz. (2003). Managing resistance to change in workplace accommodation projects. Journal of Facilities Management, 306-22.

Lara, J., Rojas, C., \& Martínez, J. (2015). Evolución organizacional: inducción sociobiológica para el entendimiento de la metáfora. AD-minister.

Lawler, \& Worley. (2006). Designig Organizations. Center of effective Organizations, 19.

Lehal, R. (2000). Managerial Creativity and Locus of Control as Determinants of Managerial Effectiveness in Public and Private Sector. Vishwavidyalaya: Indore.

Lòpez, A. M. (2008). El mundo de las empresas: De las pymes a las unimundiales. Ediciones Díaz de Santos.

Mahner, M. (2000). Fundamentos de filosofia. Mexico D.F.: Siglo Veintiuno. 
Manzano, A. (2013). Un Modelo de pensamiento estratégico para favorecer la gestión del cambio en las organizaciones. Teaching Business \& Economics, 16.

Marshak, R. (2007). Cambio Organizacional: Tabas, contatiempos y dificultades mas habituales. Barcelona: Deusto.

Martínez, E., Carrasco, C., \& Bull, M. (2018). Propuesta metodológica para implementar la primera fase del modelo de gestión del cambio organizacional de Lewin . Estudios Gerenciales.

Mazzei, A. (2010). Promoting Active Communication Behaviours Through Internal Communication, Corporate Communications. An International Journal, 221-234.

McCalman, \& James. (2008). Change Management: a guide to effective implementation. London: Sage Publications.

Mora, L., Duran, M., \& Zambrano, J. (2016). Consideraciones actuales sobre gestión empresarial. Revista cientifica donimio de las ciencias, 511-520.

Neville, \& Bell. (2005). Corporate reputation, stakeholders and the Social Performancefinancial performance relationship. European Journal of Marketing, 1184-1188.

Nohria, \& Nitin. (2000). Cracking the Code of Change. Harvard Business Review, 13 22.

O'Reilly, \& Tushman. (2008). Ambidexterity as a dynamic capability resolving the innovator's Dilemmas. Research in organizational behaviour, 185-206.

Okhuysen, G. (2001). Structuring change. Academy of Management Journal, 794-808.

Okhuysen, G. (2001). Structuring change Familiarity and formal interventions in problem solving groups. Academy of Management Journal, 794-808.

Ordóñez, R. (2010). Cambio, Creatividad e Innovación. Buenos Aires : Granica S.A.

Orozco, J. C. (2014). De Centros Cívicos a Juntas de Acción Comunal. El cambio de modelo de gestión y participación barrial en Medellín en la segunda mitad del siglo XX. Ciencia Politica, 19.

Oyarvide, H., Reyes, E., \& Montaño, M. (2017). La comunicación interna como herramienta indispensable de la administración de empresas. Dominio de las ciencias.

Paton, R., \& McCalman. (2000). Change Management. London: Sage.

Pérez, D., Hernández, D., León, E., Infante, M., Abreu, P., Ortega, Y., . . Nancy. (2017). Cuatro etapas de cambio organizacional planificado basado en las tecnologías de la información. Revista Cubana de Información en Ciencias de la Salud, 1-21. 
Pérez, L., Vilariño, C., \& Ronda, G. (2016). El cambio organizacional como herramienta para coadyuvar la implementacion de la estrategia. Ingenieria Industrial, 286-294.

Rees, \& French. (2013). Leading, Managing and Developing People.

Robbins, \& Stephen. (2012). Organizational Behaviour: Concepts, Controversies, Applications. Canada: Pearson.

Romero, J. M., \& Campo, C. (2013). Sobre el cambio organizacional. Una revisión bibliográfica. Revista de Ciencias Administrativas y Sociales.

Sala, E. M. (2001). Psicologia Evolutiva. Barcelona: anthropos.

Sanchez., E. F. (2010). Administración de empresas : un enfoque interdisciplinar. Editorial Paraninfo.

Segredo, P. (2013). Clima organizacional en la gestión del cambio para el desarrollo de la organización. Revista Cubana de Salud Pública, 386 - 397.

Senge, P. (2000). La Danza del Cambio. Colombia: Maria del Mar Ravassa.

Sidikova, \& Mashhura. (2011). THE IMPACT OF CHANGE ON EMPLOYEES ${ }^{e e}$ MOTIVATION. A CASE OF CSSC. TURKU UNIVERSITY, 70.

Srivastava, M., \& Sinha, A. (2007). Individual characteristic for managerial effectiveness in a competitive environment: an exploration.

Stohl, C. (2005). Organizational Communication: Connectedness in Action. Sage.

Szabla, D. (2007). A multidimensional view of resistance to organizational change. Human Resourse Development Quaeterly, 525-558.

Thompson, \& Strickland. (2012). Administración Estrategica. Mexico,D.F.: McGrawHill.

Tsoukas, H. (2005). Why language matters in the analysis of organizational change. Journal of Organizational Change Management, 96 -104.

Valackiene. (2010). The Expression of Effective Crisis Communication in Today's Corporation: Theoretical Insights and Practical Application. Transformations in Business \& Economics, 490-504.

Van de Ven, A. (2004). Explaining development and change in organizations. The Academy of Management Review, 847 -865.

Vecino, J. M. (2014). Hacia una cultura del cambio o hacia un cambio de cultura? Estados Unidos: Global Network Content Services LLC, DBA Noticias Financieras LLC. 
Vértiz, G. (2008). Referentes teóricos para el Análisis del Cambio Organizacional. Gestión y Estrategia, 14 - 19.

Williams, \& Williams. (2007). A change management approach to evaluating ICT investment initiatives. Journal of Enterprise Information Management, 32 -50.

Zuleta, L., Jaramillo, L., \& Reina, M. (2003). Impacto del sector cinematográfico sobre la economía colombiana: situación actual y perspectiva. Bogotá: Proimagenes.

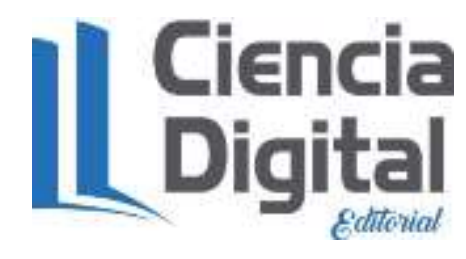




\section{PARA CITAR EL ARTÍCULO INDEXADO}

Sandoval Pérez, J. C., García Chuquimarca, M. F., Santamaría Cusco, E. J., \& Santamaría Freire, E. J. (2021). La eficiencia de la gestión del cambio en las empresas Visionario Digital, $\quad$ 5(4), $\quad$ 88-108. https://doi.org/10.33262/visionariodigital.v5i4.1915

\section{Ciencia
Digital
$\varepsilon$ thitala}

El artículo que se publica es de exclusiva responsabilidad de los autores y no necesariamente reflejan el pensamiento de la Revista Visionario Digital.

El artículo queda en propiedad de la revista y, por tanto, su publicación parcial y/o total en otro medio tiene que ser autorizado por el director de la Revista Visionario Digital.
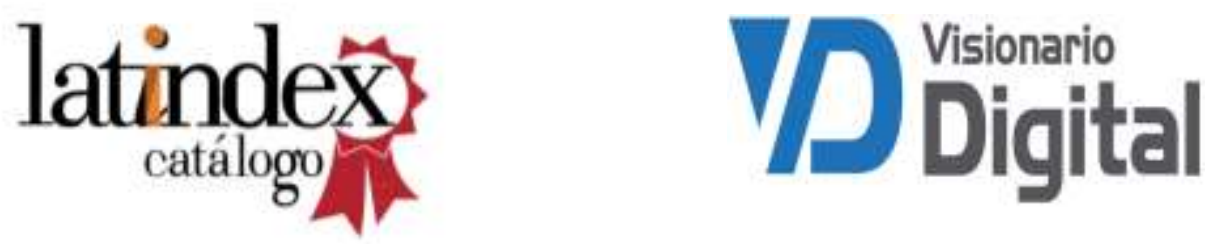